\title{
Performance Regimes of Uncoded Linear Communications over AWGN Channels
}

\author{
Szymon Jakubczak*, John Z. Sun ${ }^{\dagger}$, Dina Katabi*, Vivek K. Goyal ${ }^{\dagger}$ \\ ${ }^{*}$ Computer Science and Artificial Intelligence Laboratory ${ }^{\dagger}$ Research Laboratory of Electronics \\ Massachusetts Institute of Technology, Cambridge, MA 02139, USA \\ Email: $\{$ szym,johnsun,dk,vgoyal $\} @$ mit.edu
}

\begin{abstract}
Streaming degradable content (such as video or audio) over a wireless channel presents new challenges to modern digital design, which was founded on the separation theorem of Shannon theory. Joint source-channel coding (JSCC) has recently received increasing interest to address the varying nature of the wireless channel conditions (under mobility or multicast). The conventional approach to JSCC which combines successive refinement with superposition coding is still digital and separable. However, for a white Gaussian source on a white Gaussian channel, it is outperformed by uncoded, linear scaling, which achieves Shannon's distortion limit.

Practical degradable content sources are not white, but better approximated by multivariate / non-white Gaussian models. We investigate the performance of a linear, uncoded communication scheme for such sources. We find that there exist regimes where the uncoded scheme is near-optimal for point-to-point communication and provides significant gains over the conventional digital design for broadcast.
\end{abstract}

\section{INTRODUCTION}

For point-to-point communication, it is well known that digital encoding and decoding can provide the optimal endto-end performance with respect to a distortion metric. The separation theorem, based on ideas from Shannon [1], states that the designs of the source and channel codes can be decoupled without loss of optimality. Further, recent advances in coding theory have produced practical channel codes that approach the channel capacity [2]. As a result, today the general intuition is that digital transmission is superior to analog (or uncoded) transmission.

However, digital transmission also has some drawbacks. In particular, channel coding assumes that the channel statistics are known to the source. When this assumption is violated, i.e., mismatch exists between the target and actual channel capacity, the performance of these codes degrades sharply, showing a threshold effect. This threshold degradation is a direct result from the separation of channel and source coding, where errors at the channel decoder may render the output of the source decoder completely random. The second drawback is that the separation principle applies only to point-to-point channels and could incur a severe penalty for other channel types [3]. Last, digital communication usually requires long blocklengths and high encoder/decoder complexity to achieve near-optimal distortion.

The above limitations of digital transmission are particularly important for wireless channels. Specifically, in mobile scenarios the channel SNR may vary widely and unpredictably [4], creating a mismatch between the instantaneous channel capacity and the target capacity of the used channel code. To prevent such a situation, a digital design may need to be overly pessimistic in its choice of channel-code rate. Broadcast channels are also problematic for digital design because receivers may have diverse channel SNRs, invalidating the assumptions of the separation theorem. Finally, in sensor networks and mobile applications, latency and computational complexity become important design decisions. As a result, one may prefer a mild increase in distortion if it results in a reduction in latency or computational complexity.

To address the deficiencies of the digital design in mobile and broadcast scenarios, multiple recent papers have proposed analog or hybrid transmission schemes within a joint sourcechannel coding framework (JSCC) [5]-[8]. The advantages of such designs is that performance degrades gracefully when the channel statistics differ from their predicted values. Also, by adopting a JSCC approach they need not pay a penalty in scenarios where the separation theorem does not hold. Furthermore, some of these designs, like SoftCast [7], are fully linear and hence incur relatively low latency and computational complexity. On the negative side, however, these designs do not enjoy the aforementioned desirable properties of digital transmissions systems such as their optimality for point-topoint channels and effectiveness at compressing source data. Given this tradeoff, there is a need to accurately characterize and quantify the conditions under which an analog design may be preferable or as efficient as a digital design.

In this paper, we consider the JSCC approach underlying SoftCast, a wireless video broadcast system proposed in [7]. At the core of SoftCast lies a near-analog, uncoded linear communication scheme, to which we subsequently refer as analog. The goal of this work is to show that, for transmission of degradable sources over Gaussian channels, this scheme can achieve similar end-to-end distortion to more complex digital methods while maintaining robustness to channel variations and lowering computational complexity. In particular, we demonstrate that the digital and analog systems are comparable in performance for practical SNR regimes when the number of source components per unit time exceed the number of channel slots available. Moreover, we show the analog system intrinsically has distortion performance that degrades gracefully with channel SNR, providing substantial net performance gain for 
broadcast channels or when the channel statistics are not well estimated.

These results are a generalization of a well-known fact that an iid Gaussian source transmitted uncoded through an AWGN channel achieves optimal performance for any channel SNR [9]. Essentially, we exploit the match between the source and channel and leverage the ability to perform efficient analog compression, both factors that do not hold in general.

\section{RELATED WORK}

This paper builds on results from the fields of joint sourcechannel coding, wireless communications and video coding which exploit the fact that Gaussian sources transmitted uncoded over Gaussian channels can be optimal [9], [10]. Other combinations of sources and channels where uncoded transmission is optimal are also explored [11]. The Gaussian match was generalized to linear encoding in the vector case [12] and nonlinear encoding using space-filling curves when the number of source components does not match the number of channel uses per unit time [13], [14]. The problem of bandwidth expansion and compression has also been addressed by hybrid systems combining digital techniques with linear analog encoding [15], [16], although the scope of analysis was limited to a 2-dimensional white source. Correlation in the source was shown to aid the broadcast scenario in a hybrid scheme proposed in [17].

Of particular interest are investigations of analog or hybrid systems that transmit media content over AWGN channels. In [5], wireless image transmission is made more robust using an analog transmitter. In [6], a nonlinear analog image encoder is designed to achieve graceful degradation of quality with channel SNR. In [7], a practical system called SoftCast uses linear encoding to achieve robustness to channel variations for video broadcast.

Also related to this work are predominantly digital schemes that perform JSCC to alleviate some of issues with digital communication discussed previously. In [18], multiresolution coding with many refinement layers allows for degradation of performance to vary with channel SNR like in analog coding.

There are also purely digital systems that try to address robustness for wireless communications and broadcast of media. Proposals for scalable video communications in broadcast wireless channels combine successive refinement in a layered source coder, such as h.264/SVC [19] with hierarchical modulation [20]. Other proposals operate at a fine granularity by employing unequal bit-error protection [21], [22].

\section{PRELIMINARIES}

We present our problem model and review basic results for communication, both digital and analog, of Gaussian sources over Gaussian channels. We also introduce the analog transmission scheme used later in the paper. For this section, we limit the exposition to the major concepts rather than the formal definition and proofs which can be found in [23].

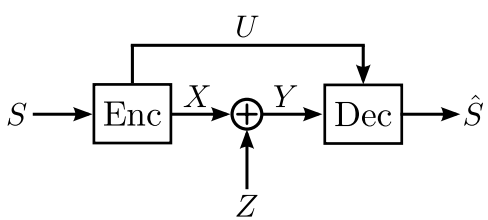

Fig. 1: A system model for communications. A side channel may exist between the encoder and decoder to transmit a small amount of metadata to aid signal reconstruction.

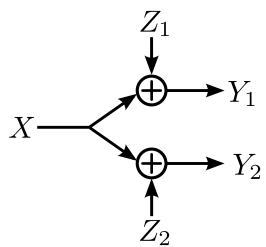

Fig. 2: The two-user broadcast channel model. The transmission $X$ is desired by both users but is corrupted by noise.

\section{A. Problem Statement}

Consider the system depicted in Figure 1. At each time step, the encoder accepts a vector $S \in \mathbb{R}^{N}$ and transmits a vector $X \in \mathbb{R}^{M}$ over a set of parallel channels perturbed by an additive white Gaussian noise (AWGN) vector $Z$. The decoder receives the noisy transmission $Y=X+Z$ and produces a reproduction $\hat{S}$ of the transmitted signal. The goal of the system is to minimize the distortion between $S$ and $\hat{S}$, measured as the mean-squared error (MSE), i.e., $D=E\left[\sum_{i}\left(\hat{S}_{i}-S_{i}\right)^{2}\right]$. The constraint is on the the average power of the encoder, meaning

$$
E\left[\frac{1}{M} \sum_{i} X_{i}^{2}\right] \leq P .
$$

For this paper, we assume the source $S$ is the class of memoryless multi-variate Gaussian vectors with diagonal covariance matrices. Hence, each component or subband $S_{i}$ of the source is an independent zero-mean Gaussian variable with variance $\lambda_{i}$. Assume a natural order of subbands according to variances such that $\lambda_{1} \geq \cdots \geq \lambda_{N}$. Moreover, assume the noise components $Z_{i}$ are iid with variance $\sigma^{2}$. The channel can also be characterized using a signal-to-noise ratio $\mathrm{SNR}=P / \sigma^{2}$.

Section V discusses a two-user broadcast channel as shown in Figure 2. For this system, each user may have different channel statistics. We define the noise variances as $\sigma_{1}^{2}$ and $\sigma_{2}^{2}$ (corresponding to $\mathrm{SNR}_{1}$ and $\mathrm{SNR}_{2}$ ) and assume $\sigma_{1} \leq \sigma_{2}$.

\section{B. Digital Communication}

The capacity $C(\mathrm{SNR})$ of the channel defines the maximum rate of the bit-stream that can be decoded without error for a given channel SNR. Meanwhile, the rate-distortion function of the source, $R(D)$ defines the minimum achievable rate $R$ that yields distortion $D$. Hence $R\left(D_{\text {opt }}\right)=C(\mathrm{SNR})$. This is also known as OPTA (Optimum Performance Theoretically Attainable). Note that achieving an end-to-end distortion of $D_{\text {opt }}$ could require infinite block length in both source and channel coders. 
For a set of $M$ AWGN channels with a particular SNR, the capacity is

$$
C(\mathrm{SNR})=\frac{M}{2} \log (1+\mathrm{SNR}) .
$$

For a multi-variate Gaussian $S$, the rate-distortion function is given by the reverse water-filling solution:

$$
R(D)=\sum_{i=1}^{N} \frac{1}{2} \log _{2} \frac{\lambda_{i}}{D_{i}}
$$

where $D_{i}=\min \left(\gamma, \lambda_{i}\right)$ and $\gamma$ is chosen to satisfy $\sum_{i} D_{i}=D$.

By reverse water-filling, some source subbands may not be transmitted when the channel is noisy. For a given source $S$ and channel SNR, we can find the optimal choice of $\gamma$ and subsequently $K$, the number of subbands transmitted. The resulting optimal end-to-end distortion is

$$
D_{\mathrm{dig}}(\mathrm{SNR})=K\left(\frac{\prod_{i=1}^{K} \lambda_{i}}{(1+\mathrm{SNR})^{M}}\right)^{1 / K}+\sum_{i=K+1}^{N} \lambda_{i} .
$$

We also survey results related to the Gaussian broadcast channel model of Figure 2, where the capacity is known since the channel is stochastically degraded, i.e., can be modeled as $Y_{2}=X+Z_{2}=Y_{1}+Z_{2}^{\prime}$, where $\sigma_{2}^{\prime 2}=\sigma_{2}^{2}-\sigma_{1}^{2}$ [24]. The achievable rate region can be obtained by superposition coding, where the encoder splits the bit-stream into lowpriority enhancement at rate $R_{1}$ and high-priority base at rate $R_{2}$, channel-codes them, and adds them up before transmitting. Both users can decode the base and the stronger receiver can also decode the enhancement. The achievable rate region is given by

$$
\begin{aligned}
& R_{1}=\frac{M}{2} \log _{2}\left(1+\omega \mathrm{SNR}_{1}\right), \\
& R_{2}=\frac{M}{2} \log _{2}\left(1+\frac{(1-\omega) \mathrm{SNR}_{2}}{\omega \mathrm{SNR}_{2}+1}\right),
\end{aligned}
$$

where $\omega \in[0,1]$ is a parameter that determines the fraction of total power devoted to transmitting the enhancement. Superposition coding in QAM is also known as hierarchical modulation.

The matching source coder needs to encode the source into two bit-streams. For example, the high-priority base is result of a coarse quantization, and the low-priority enhancement is a finer quantization of the residual error from the coarse quantization. Gaussian sources are successively refinable without loss, and therefore the two users can achieve, respectively, distortions $D_{1}=D\left(R_{1}+R_{2}\right)$ and $D_{2}=D\left(R_{2}\right)$, i.e., the minimum distortion achievable at the total received rate. Note that for two users, a two-layer digital scheme is maximal, i.e., cannot be improved by adding layers.

\section{Analog Communication}

For a single-dimensional Gaussian source and channel, we find that the minimum distortion

$$
D_{\text {opt }}=\lambda 2^{-2 C}=\frac{\lambda}{1+\mathrm{SNR}}
$$

is achieved by a very simple uncoded (unit block length) system:

$$
\begin{aligned}
X & =g S, \\
\hat{S} & =\frac{g \lambda}{g^{2} \lambda+\sigma^{2}} Y .
\end{aligned}
$$

The encoder applies a scaling factor $g=\sqrt{P / \lambda}$ to match available power, and the decoder performs linear least squares (LLS) estimation given the known noise variance. We assume that the statistic $\lambda$ is communicated to the decoder via a side channel. Such an encoder is often referred to in the literature as Pulse Amplitude Modulation (PAM).

In this paper, we consider a generalization of this analog system with the restriction that encoding and decoding are linear and allow for a side channel that communicates the subband variances as side information to the decoder. The encoder transmits $X=G S$ where $G$ is an $M \times N$ matrix. Given a constraint on average power and the fact that we consider only the regime $M \leq N$, the optimal gain matrix $G$ is diagonal (for $M<N$, the $N-M$ subbands with smallest variances are suppressed). Since the LLS decoder, optimal for linear decoders, depends on noise variance, the encoder can optimize the gain matrix expecting a specific channel SNR. Denoting the diagonal elements of $G$ to be $g_{i}$ and the normalized gain $d_{i}=g_{i}^{2} / P$, the distortion of the analog system can be written as

$$
D(\mathrm{SNR})=\sum_{i=1}^{N} \frac{\lambda_{i}}{d_{i} \lambda_{i} \mathrm{SNR}+1}
$$

subject to $\sum_{i} d_{i} \lambda_{i}=M$ and $d_{i} \geq 0$. This yields an analog water-filling result [12]:

$$
D_{\text {ana }}(\mathrm{SNR})=\frac{\left(\sum_{i=1}^{K} \sqrt{\lambda_{i}}\right)^{2}}{M \mathrm{SNR}+K}+\sum_{i=K+1}^{N} \lambda_{i},
$$

where the number of subbands to be transmitted, $K$, is dependent on the channel SNR, much like in the digital case. The choice of $K$ can be determined by solving a Lagrangian and increases monotonically with SNR up to $M$.

We briefly comment on the robustness of this analog scheme. Because the system is linear, a mismatch between design and actual SNR does not necessarily induce complete performance loss like in the digital case when coding near capacity. In fact, for reasonable mismatches, the performance may still be near optimal for the actual SNR. A rigorous analysis of robustness for the single-component case is given in [25].

\section{Point-to-Point Communication}

We compare the end-to-end distortion performance between the proposed analog system and a digital system operating at capacity using the model in Section III. We define the compression ratio to be $\beta=M / N$ and the performance ratio to be $\rho\left(\mathrm{SNR}, \Lambda_{S}\right)=D_{\operatorname{dig}} / D_{\text {ana }}$, where $\Lambda_{S}$ is the covariance matrix of the source and $D_{\mathrm{dig}}$ and $D_{\text {ana }}$ are the solutions to (3) and (10) for a target channel SNR. 


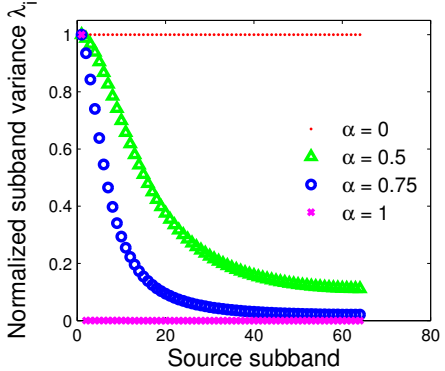

Fig. 3: Plot of normalized subband variance $\lambda_{i}$ for selected values of source correlation parameter $\alpha$.

We consider the case when $N$ and $M$ are arbitrary but $M \leq$ $N$ holds. In this paper, we omit the case of $\beta>1$ because it has been well-studied that linear analog encoding performs poorly compared to nonlinear coding such as space-filling [13] in this regime.

Since the parameter space can be arbitrarily large, we restrict the source to a first-order Gauss-Markov (or AR(1)) process that is decorrelated using the Karhunen-Loève Transform (KLT) to form the vector $S$. We emphasize that the qualitative results hold more generally and this model is a tool to reduce the parameter space. Moreover, this model has strong ties to media compression due to the fact that the KLT and DCT are asymptotically equivalent [26].

We assume a source $S_{\mathrm{GM}}(t) \in \mathbb{R}^{N}$ is independent in time but its components at a given instant form a stationary Gaussian process with a covariance matrix

$$
\left(\Lambda_{\mathrm{GM}}\right)_{i j}=\alpha^{|i-j|}, \quad i, j=1,2, \ldots N .
$$

The KLT of $\Lambda_{\mathrm{GM}}$ is a diagonal matrix $\Lambda_{S}$ with entries corresponding to the eigenvalues of $\Lambda_{\mathrm{GM}}$. They can be expressed as

$$
\lambda_{i}=\frac{1-\alpha^{2}}{1-2 \alpha \cos \theta_{i}+\alpha^{2}},
$$

where the $\theta_{i}$ s are the positive roots of

$$
\tan (N \theta)=-\frac{\left(1-\alpha^{2}\right) \sin \theta}{\cos \theta-2 \alpha+\alpha^{2} \cos \theta} .
$$

These eigenvalues can be seem as taking non-uniform samples from the monotonically decreasing function

$$
f(\theta ; \alpha)=\frac{1-\alpha^{2}}{1-2 \alpha \cos (\theta)+\alpha^{2}}
$$

in the range $[0, \pi)$ [27]. Hence, the input to the encoder is $S$, a multi-variate memoryless Gaussian vector with decaying variances assumed in earlier sections. For reference, Figure 3 shows how $\alpha$ affects the decay of $\lambda$.

When $M=N$, the performance results are well known. In the degenerate case of $\alpha=0$, the system has no memory and this problem simplifies to that of an iid Gaussian source through AWGN channel with $\rho=1$. For general $\alpha$ and high $\mathrm{SNR}$, the ratio approaches the constant

$$
\rho=N^{2} \frac{\left(\prod_{i=1}^{N} \lambda_{i}\right)^{1 / N}}{\left(\sum_{i=1}^{N} \sqrt{\lambda_{i}}\right)^{2}}
$$

which is the Arithmetic Mean-Geometric Mean performance gap discussed in [5].

It is also well-known that $\rho \approx 1$ for very low SNR in general. It may be tempting to say that for $\beta<1$, these same trends hold. However, we show this is not the case and $\rho$ does not necessarily behave in a monotonic fashion with respect to SNR. At one extreme, using the distortion results (3) and (10), we see that for high SNR, $\rho$ decays as $(1+\mathrm{SNR})^{\beta}$ and hence the performance loss is dramatic. This is because a digital scheme can successfully compress $N$ source symbols into $M$ channel uses but an analog design must discard the $N-M$ components with smallest variances, which is inefficient. Meanwhile, for moderate SNR, we note that both analog and digital transmission may not transmit all source components possible. When the analog and digital transmit both communicate approximately $M$ components, $\rho$ can be close to 1 . In this case, the analog scheme remains efficient because it is transmitting as many components as it can support with $M$ channel uses while the digital is forced to suppress some subbands due to the noisiness of the channel, thereby reducing its asymptotic advantage.

This is demonstrated in Figure 4, which looks at several choices of $M$ for $N=64$. We can note that for smaller $\beta$ and moderate SNR, the analog design performs much better than asymptotic analysis may suggest. These results show that an analog design is reasonable beyond just the iid Gaussian case and the Arithmetic Mean-Geometric Mean performance gap does not hold except for $\beta=1$. A designer may tolerate such performance loss in favor of the simplicity and robustness of the analog system.

\section{BROADCAST COMMUNICATION}

We now consider the degraded broadcast case and compare the ideal purely-digital system (which combines successive refinement with superposition coding) and the analog linear system using the model in Section III. The end-to-end distortion performance is defined in terms of the distortions observed by each of the users and depends on channel SNR of each user. To comparatively evaluate the two systems, we use total distortion, i.e., sum of distortions of individual users. In general, one could use other metrics, e.g., the weighted sum. This overall distortion metric is a function of each user's channel SNR. For simplicity, we consider a scenario with only two users, and focus on three operational SNR regimes:

1) high SNR: $\mathrm{SNR}_{1}=17 \mathrm{~dB}$ and $\mathrm{SNR}_{2}=10 \mathrm{~dB}$

2) low SNR: $\mathrm{SNR}_{1}=10 \mathrm{~dB}$ and $\mathrm{SNR}_{2}=3 \mathrm{~dB}$

3) wide SNR: $\mathrm{SNR}_{1}=17 \mathrm{~dB}$ and $\mathrm{SNR}_{2}=3 \mathrm{~dB}$

As in the case of point-to-point, one parameter in the evaluation is compression ratio, $\beta=M / N$. However, we also consider the case of bandwidth expansion, i.e., $\beta>1$. Although the linear analog scheme cannot compete in this regime for point-to-point channels except for very low SNR, the broadcast setting gives it advantages over the digital system.

Thus the computed performance ratio is $\rho\left(\mathrm{SNR}_{1,2}, \Lambda_{S}\right)=$ $D_{\text {dig }} / D_{\text {ana }}$, where $\Lambda_{S}$ is the covariance matrix of the source 


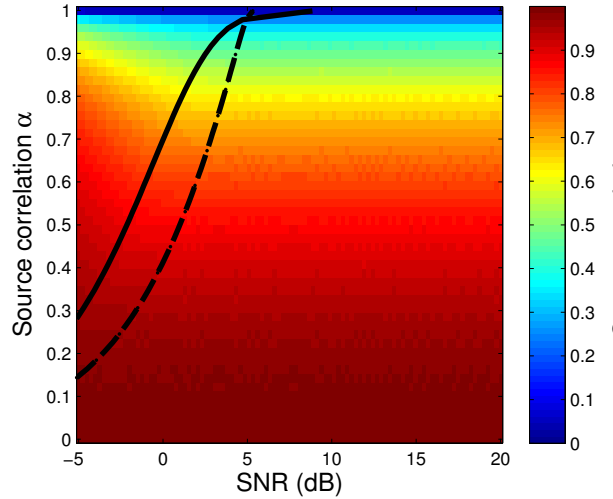

(a) $M=64$

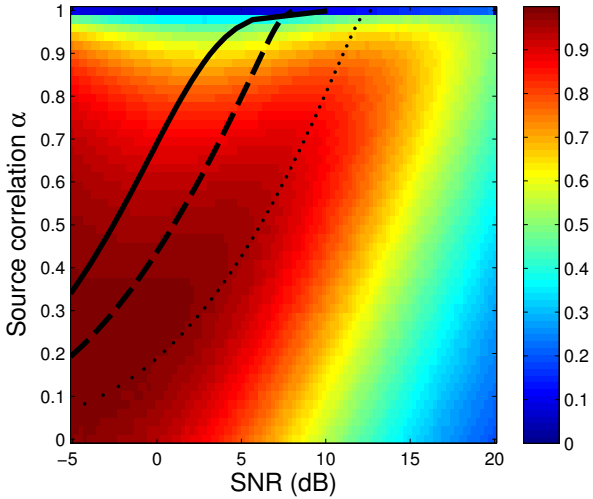

(b) $M=32$

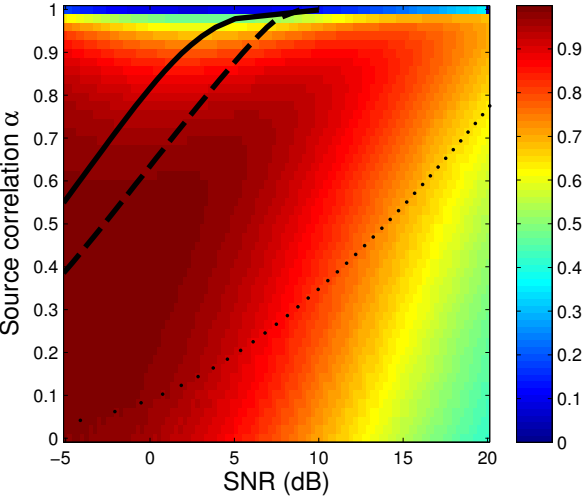

(c) $M=16$

Fig. 4: Plot of performance ratio $\rho$ for $N=64$ in terms of $\alpha$ and SNR in point-to-point communication. The solid line corresponds to the threshold for when the analog scheme transmits $M$ subbands. The dashed and dotted lines correspond to the thresholds for when the digital scheme transmits $M$ and $N$ source subbands respectively.

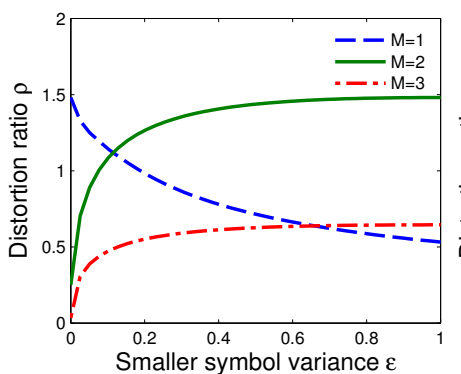

(a) $\mathrm{SNR}_{1}=17 \mathrm{~dB}, \mathrm{SNR}_{2}=10 \mathrm{~dB}$

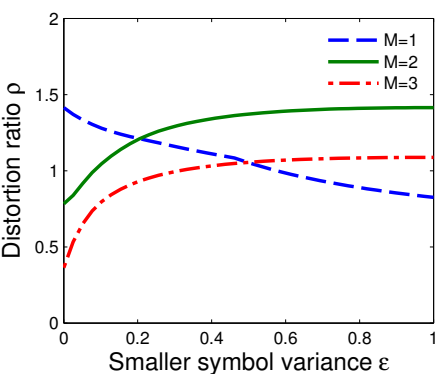

(b) $\mathrm{SNR}_{1}=10 \mathrm{~dB}, \mathrm{SNR}_{2}=3 \mathrm{~dB}$

Fig. 5: Broadcast performance ratio $\rho$ for the 2-dimensional source in terms of the smaller subband variance $\epsilon$ and channel bandwidth $M$. The three lines correspond to the case of bandwidth compression, match, and expansion.

and $D_{\text {dig }}$ and $D_{\text {ana }}$ are the minimum total distortions achievable in either scheme when optimized over its tunable parameters according to Section III. That is, in both cases the encoder decides how many source subbands to suppress and the digital superposition encoder optimizes the power distribution between the two bit-streams.

\section{A. A Two-dimensional Source}

We first use a simple example to provide some intuition about how $\rho$ may vary with the source covariance matrix. Consider the case of $N=2$ and $M=1,2,3$. As before, we assume $\lambda_{1}=1, \lambda_{2}=\epsilon$.

Figure 5 plots the performance ratio $\rho$ in terms of $\epsilon$ and the channel bandwidth $M$. We observe that how $\rho$ changes with $\epsilon$ depends on the compression ratio. In the regime of bandwidth compression, when $M=1$, the analog system delivers a performance gain for low $\epsilon$, but a significant loss for white sources. In contrast, in the case of matched bandwidth $M=2$, the gain of the analog system peaks at $\epsilon=1$, as it converges to the optimal distortion bound. In the regime of bandwidth expansion, $M=3$, the analog system, limited by its linearity, loses its broadcast advantage. The lower the $\epsilon$, the

worse performance it exhibits.

This points at the underlying determining factor of analog performance. When the source and channel are matched, it performs nearly optimally, but fails competitively to expand or compress the bandwidth. As $\epsilon$ tends to 0 , the source can be approximated as single-dimensional by ignoring the lowvariance subband. Thus, for $M=1$ the system becomes matched. The performance gap (both loss and benefit) is more pronounced in the high SNR regime.

\section{B. A More General Case}

We now move to larger source dimensions, specifically $N=64$. Using the same Gauss-Markov model as in Section IV, we evaluate the performance ratio $\rho$ under varying source correlation $\alpha$ and channel bandwidth $M$. Once again, we look at a wide spectrum from bandwidth compression of $1 / 64$, to bandwidth expansion of 2 .

Figure 6 shows the performance ratio in the three considered SNR scenarios. We find that analog dominance is most pronounced in the area of matched bandwidth for low source correlation. Indeed, when the source is white $(\alpha=0)$, we expect the matched analog system to perform optimally, as discussed previously. However, we also find that analog remains dominant in the area of moderate compression and source correlation (top left in the plot). This aligns with our previous observations: if the source is correlated it can be "easily" compressed into the available channel bandwidth. The more correlated the source is, the lower the matching compression ratio.

We find that, although the analog system shows the greatest maximum benefit in the high SNR regime, its performance gain decays abruptly with source-channel rate mismatch. On the other hand, if the SNR of the weak user is low, the analog system is superior in a larger domain. The modest difference between Fig. $6 \mathrm{~b}$ and $6 \mathrm{c}$ suggests that the dominating factor is the lower SNR rather than the SNR range. However, the overall benefit of the analog is generally diminished at higher 


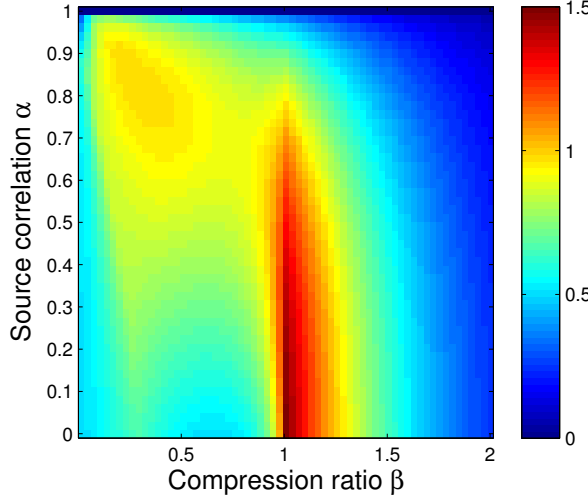

(a) $\mathrm{SNR}=\{17,10\} \mathrm{dB}$

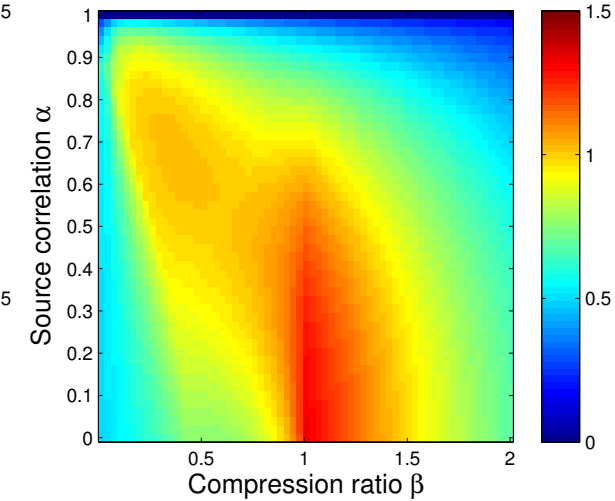

(b) $\mathrm{SNR}=\{17,3\} \mathrm{dB}$

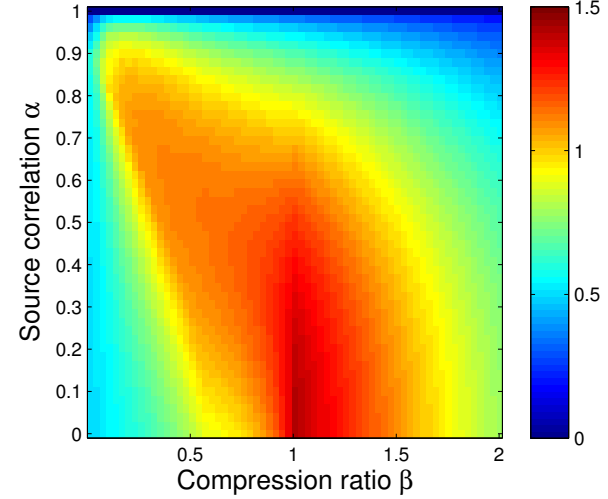

(c) $\mathrm{SNR}=\{10,3\} \mathrm{dB}$

Fig. 6: Broadcast performance ratio $\rho$ for the three SNR regimes in terms of source correlation, $\alpha$ and compression ratio, $\beta$.

SNR by the asymptotic gap. These results show that an analog design might offer substantial benefit in a broadcast scenario beyond the matched iid Gaussian case.

\section{CONCLUDing REMARKS}

In this work, we demonstrate that an analog scheme with linear encoding and decoding can be an efficient way to communicate Gaussian sources in terms of distortion, latency, robustness and computational complexity. We show that the distortion performance of an analog system is comparable to that of its digital counterpart for many regimes of practical interest in the point-to-point case and demonstrate additional benefits in a broadcast setting. We also discuss how this can be useful in a variety of applications where the content is degradable and connect our findings to practical schemes that are easily implementable in hardware. We believe that there is still value to designing analog systems for certain sourcechannel models and there is a significant amount of interesting future work to be done in this area.

\section{REFERENCES}

[1] C. E. Shannon, "A mathematical theory of communication," Bell Syst. Tech. J., vol. 27, pp. 379-423, Jul. 1948, continued 27:623-656, October 1948.

[2] D. MacKay and R. Neal, "Near Shannon limit performance of low density parity check codes," Electronics letters, vol. 33, no. 6, pp. 457458, 1997.

[3] M. Gastpar, M. Vetterli, and P. L. Dragotti, "Sensing reality and communicating bits: A dangerous liaison," IEEE Sig. Process. Mag., vol. 23, no. 4, pp. 70-83, Jul. 2006.

[4] M. Vutukuru, H. Balakrishnan, and K. Jamieson, "Cross-layer wireless bit rate adaptation," SIGCOMM Comput. Commun. Rev., vol. 39, no. 4, pp. 3-14, 2009.

[5] I. Kozintsev and K. Ramchandran, "A wavelet zerotree-based hybrid compressed/uncompressed framework for wireless image transmission," in 31st Asilomar Conf. Sig., Sys., \& Computers, Nov. 1997.

[6] H. Coward, "Joint source-channel coding: Development of methods and utilization in image communications," Ph.D. dissertation, Norwegian University of Science and Technology, 2002.

[7] S. Jakubczak, H. Rahul, and D. Katabi, "One-size-fits-all wireless video," in HotNets, 2009.

[8] N. Geri, "Wireless uncompressed hdtv solves problems that compression cannot," EE Times, May 2007.

[9] T. J. Goblick, Jr., "Theoretical limitations on the transmission of data from analog sources," IEEE Trans. Inform. Theory, vol. IT-11, no. 4, pp. 558-567, Oct. 1965.
[10] T. Berger, Rate Distortion Theory. Englewood Cliffs, NJ: Prentice-Hall, 1971.

[11] M. Gastpar, B. Rimoldi, and M. Vetterli, "To code, or not to code: Lossy source-channel communication revisited," IEEE Trans. Inform. Theory, vol. 49, no. 5, pp. 1147-1158, 2003.

[12] K.-H. Lee and D. P. Petersen, "Optimal linear coding for vector channels," IEEE Trans. Communications, Dec 1976.

[13] S. Chung, "On the construction of some capacity-approaching coding schemes," Ph.D. dissertation, Citeseer, 2000.

[14] Y. Hu, J. Garcia-Frias, and M. Lamarca, "Analog Joint Source Channel Coding Using Space-Filling Curves and MMSE Decoding," in Data Compression Conference. IEEE Computer Society, 2009, pp. 103-112.

[15] M. Skoglund, N. Phamdo, and F. Alajaji, "Hybrid digital-analog sourcechannel coding for bandwidth compression/expansion," IEEE Trans. Information Theory, vol. 52, no. 8, pp. 3757-3763, 2006.

[16] Z. Reznic, M. Feder, and R. Zamir, "Distortion bounds for broadcasting with bandwidth expansion," IEEE Trans. Information Theory, vol. 52, no. 8, pp. 3778-3788, Aug. 2006.

[17] V. Prabhakaran, R. Puri, and K. Ramchandran, "Hybrid digital-analog strategies for source-channel broadcast," in Allerton Conference, 2005.

[18] K. Ramchandran, A. Ortega, M. Uz, and M. Vetterli, "Multiresolution broadcast for digital HDTV using joint source/channel coding," IEEE J. Sel. Areas Comm., vol. 11, no. 1, pp. 6-23, Jan. 1993.

[19] H. Schwarz, D. Marpe, and T. Wiegand, "Overview of the scalable video coding extension of the H.264/AVC standard," in IEEE International Symposium on Circuits and Systems (ISCAS), 2007.

[20] T. Kratochvíl, Hierarchical Modulation in DVB-T/H Mobile TV Transmission, 2009, pp. 333-341.

[21] A. B. Abdurrhman, M. E. Woodward, and V. Theodorakopoulos, Robust Transmission of H.264/AVC Video Using 64-QAM and Unequal Error Protection, 2009, pp. 117-125.

[22] X. Xu, M. van der Schaar, S. Krishnamachari, S. Choi, and Y. Wang, "Fine-granular-scalability video streaming over wireless lans using cross layer error control," in IEEE ICASSP, vol. 5, May 2004.

[23] T. Cover and J. Thomas, Elements of Information Theory, 1991.

[24] T. M. Cover, "Broadcast channels," IEEE Trans. Inform. Theory, vol. 18, no. 1, pp. 2-14, Jan. 1972.

[25] L. R. Varshney, "Optimal information storage: Nonsequential sources and neural channels," Master's thesis, Mass. Inst. Tech., Jun. 2006.

[26] K. R. Rao and P. Yip, Discrete Cosine Transform: Algorithms, Advantages, Applications. San Diego, CA: Academic Press, 1990.

[27] W. D. Ray and R. M. Driver, "Further decomposition of the KarhunenLoève series representation of a stationary random process," IEEE Trans. Inform. Theory, vol. IT-16, no. 6, pp. 663-668, Nov. 1970. 